\title{
The palmomental reflex: stop scratching around!
}

\author{
Jonathan M Schott MD FRCP, Martin N Rossor MD FMedSci
}

Dementia Research Centre, UCL Institute of Neurology, Queen Square, London WC1N 3BG j.schott@ucl.ac.uk

550 words

6 references

The palmomental reflex is considered to be one of the frontal release signs, also known as primitive reflexes. [1] These reflexes include utilisation behaviour, grasping, rooting, sucking and snouting, but not pouting - any reflex involving a strike is a myotactic stretch reflex and is indicative of an upper motor neurone lesion. [2] Present in some mammals where they may confer some some survival benefits, these reflexes are thought to be present early in human development, to be supressed during cortical maturation, but to re-emerge in the presence of neurological disease affecting the frontal lobes. Typically elicited by gently scratching an orange stick from the proximal to distal aspect of the thenar eminence, a positive palmomental reflex is when there is a single synchronous contraction of the ipsilateral mentalis muscle, i.e. a twitch at the chin. [3]

At face value, the palmomental reflex is perhaps the perfect stereotype of the neurologist's art: visually arresting and perfect for grand rounds or to impress medical students; obscure (who else but a neurologist will have ever heard of it?); perhaps explicable on neuroanatomical grounds - although its exact neurophysiological underpinnings remain uncertain [3][4]; and providing a simple bedside means of uncovering focal pathology in the frontal lobes, a cortical region where "hard" neurological signs are notoriously elusive. The only problem is that it is more often than not completely useless.

First, whilst there is no real consensus as to how exactly it should be elicited (with a key? with an orange stick? with light or deep pressure? once or repeatedly?), [3] more often than not it is definitely elicited incorrectly: the authors have witnessed all kinds of fervent palm scratching accompanied by varying degrees of facial grimacing in the testing junior doctorif not the patient (Figure). Second, neurophysiological studies have confirmed that it is a normal phenomenon: sufficient electrical stimulus of the palm produces a mentalis response in all subjects [5]. Whilst the degree of stimulation required to elicit a mentalis response, or extent of fatigability observed with repeat testing may (or may not) differ between controls and patients [3], in practice the palmomental reflex even when elicited at the bedside cannot therefore be considered a sensitive marker for disease, with studies quoting it to be present in as many as half of healthy people. [3][6] Fourth, even when present in the context of a disease state it has no specificity for any one pathology, being seen in numerous and varied conditions including Alzheimer's disease, cerebrovascular pathology, Down's syndrome [3], mass lesions and hydrocephalus [4]. And finally, it has limited if any localising value - there is no clear association between the side the reflex is elicited and the side the facial twitch is observe - indeed there may be bilateral contractions; nor does its presence even imply that the pathology is restricted to the frontal 
lobe, being seen in the context of focal lesions elsewhere, and in a range of neurodegenerative and developmental disorders [3][4]

However clever and quick to administer, an inconsistently administered test lacking sensitivity, specificity or localising value has no place in modern neurological clinical practice. Whilst subject to considered critique - and indeed detailed review [3] - over many years, the palmomental reflex remains remarkably recalcitrant. It is time to put it out of its misery, and abandon it once and for all.

\section{References}

1. Schott JM, Rossor MN. The grasp and other primitive reflexes. J Neurol Neurosurg Psychiatry. 2003;74(5):558-60.

2. Rossor MN. Snouting, pouting and rooting. Practical Neurology, 2001:119-121

3. Owen, Mulley GP. The palmomental reflex: a useful clinical sign? J Neurol Neurosurg Psychiatry 2002;73:113-115

4. Gotkine M, Haggiag S, Abramsky O, Biran I. Lack of hemispheric localizing value of the palmomental reflex. Neurology 2005;64(9):1656.

5. Reis DJ. The palmomental reflex. A fragment of a general nociceptive skin reflex: a physiological study in normal man. Arch Neurol 1961;4:30-42.

6. Jacobs L, Gossman MD. Three primitive reflexes in normal adults. Neurology 1980;30(2):184 Fe

Provenance and peer review. Commissioned. Externally peer reviewed.

This paper was reviewed by Marty Samuels, Boston, USA and Martin Turner, Oxford, UK. Dr Schott write the first draft which was reviewed by Professor Rossor. Both authors agreed the final draft. Funding - none; Competing interests - none.

\section{Referee's comment}

I also find the palmomental reflex useful only for roundsmanship. Like any of the so-called frontal release reflexes (those that are present in early life, but are suppressed by development) they are found in otherwise normal people and rarely help in determining the localization and etiology for the problem. However, sometimes a very exaggerated release reflex does point to a disease of the cerebrum, though it can be frontal or elsewhere and also structural or functional. For example, visual sucking is probably always pathological, even in the very elderly patient, though it does not localize the lesion. Self grasping is nearly always abnormal, but it too suffers from lack of accurate localizing value. Martin A Samuels. 